Dominant role of many-body effects on the carrier distribution function of quantum dot lasers

This content has been downloaded from IOPscience. Please scroll down to see the full text.

2016 Appl. Phys. Express 9032705

(http://iopscience.iop.org/1882-0786/9/3/032705)

View the table of contents for this issue, or go to the journal homepage for more

Download details:

IP Address: 130.209.115.82

This content was downloaded on 30/03/2016 at $16: 28$

Please note that terms and conditions apply. 


\title{
Dominant role of many-body effects on the carrier distribution function of quantum dot lasers
}

\author{
Negin Peyvast, Kejia Zhou, Richard A. Hogg, and David T. D. Childs \\ Department of Electronic and Electrical Engineering, University of Sheffield, Centre for Nanoscience and Technology, \\ North Campus, Sheffield S3 7HQ, U.K.
}

Received December 18, 2015; accepted February 1, 2016; published online February 18, 2016

The effects of free-carrier-induced shift and broadening on the carrier distribution function are studied considering different extreme cases for carrier statistics (Fermi-Dirac and random carrier distributions) as well as quantum dot (QD) ensemble inhomogeneity and state separation using a Monte Carlo model. Using this model, we show that the dominant factor determining the carrier distribution function is the free carrier effects and not the choice of carrier statistics. By using empirical values of the free-carrier-induced shift and broadening, good agreement is obtained with experimental data of QD materials obtained under electrical injection for both extreme cases of carrier statistics.

(C) 2016 The Japan Society of Applied Physics

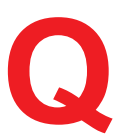
uantum dot (QD) lasers have attracted significant interest for more than thirty years owing to the prediction of temperature insensitivity ${ }^{1)}$ and enhanced material gain. ${ }^{2}$ ) The Stranski-Krastanov growth method has been employed to realise high-quality laser devices $^{3)}$ and has been used to extend the wavelength of GaAs-based lasers to $1300 \mathrm{~nm}^{4)}$ and beyond. ${ }^{5)}$

The importance of the carrier distribution within semiconductor laser materials has been highlighted and discussed widely, ${ }^{6-10)}$ and can be expected to play a key role in many aspects of the operation of the laser diode.

GaAs-based InAs QDs operating at $\sim 1000 \mathrm{~nm}$ have been shown to exhibit a carrier distribution in thermal equilibrium at $300 \mathrm{~K},{ }^{6}$ but a breakdown in this thermal equilibrium was observed at low temperatures, which was attributed to the transition to a random carrier population of isolated quantum dots. ${ }^{7)}$ The details of the carrier distribution were deduced by taking the ratio of the gain $(g)$ to spontaneous emission $(\mathrm{SE})$ rate $\left(R_{\mathrm{sp}}\right)$ to determine the carrier distribution function $P_{\mathrm{f}} \cdot{ }^{6)}$

$$
P_{\mathrm{m}}=\frac{g}{R_{\mathrm{sp}}}=\frac{\pi^{2} \hbar^{3} c^{2}}{n^{2} E_{h \nu}^{2}} P_{\mathrm{f}}
$$

Under steady-state conditions, the gain and emission characteristics are linked via the electrical carrier energy distribution. Therefore, from this equation, the electron and hole population statistics can be described. For much more deeply confined GaAs/InAs QDs operating at $1300 \mathrm{~nm}$, a thermalised (Fermi-Dirac) carrier distribution at room temperature has been deduced by the analysis of the same function. ${ }^{9)}$ However, finite carrier capture and relaxation times within the quantum dots have been pointed out to have a significant impact on the carrier distribution, and that a non-thermalised carrier population is required to explain the appearance of multistate lasing from the QD ensemble. ${ }^{10)}$ The analysis of QD laser threshold current densities has suggested an intermediate case between Fermi-Dirac and random populations. ${ }^{7)}$ In all the aforementioned reports, free carrier effects in the QDs were not considered.

Free carrier effects have been shown to shift the gain peak in the QD ensemble ${ }^{11)}$ and give rise to a negative differential gain at high current densities owing to gain saturation, yet with continually increasing dephasing effects. ${ }^{12)}$

We previously reported a Monte Carlo model incorporating many-body effects for determining the gain and spontaneous emission spectra of quantum dot laser materials. ${ }^{13)}$
Our method contrasts with previous methods that consider an ensemble of identical quantum dots, assume a FermiDirac carrier distribution, and explicitly calculate quantum effects such as carrier-carrier and carrier-phonon correlations. Previous methods have dealt with QD inhomogeneity through the statistical averaging of the resultant gain spectrum. ${ }^{14)}$ This approach has been extended to investigate the effect of the position/clustering of indium atoms in QDs of fixed average composition with regard to a negative differential gain. ${ }^{15)}$ Fermi-Dirac carrier statistics were again assumed a priori. This approach accommodates some aspects of inhomogeneity present in self-assembled QD ensembles, but has been so far limited in application.

The procedure we adopt ${ }^{13)}$ is shown schematically in Fig. 1. We consider an ensemble of QDs with a Gaussian envelope for a ground-state energy distribution with a FWHM representing the inhomogeneous distributions of QD size and composition. The ground-state and excited-state (GS-ES) separation for all the QDs is assumed to be constant [see Fig. 1(a)].

In the next step [Fig. 1(b)], carrier statistics are applied to the ensemble of QD states. In the case of Fermi-Dirac statistics, a Fermi probability distribution has been used to fill the QD states to a required average QD occupancy. In the case of a random carrier distribution, the QDs are filled independent of their energy and occupation to the required average QD occupancy. Assumptions considered in this approach are that we have a geminate population with charge neutral QDs in both cases. The microstate of the $\mathrm{QD}^{16}$ ) is determined by considering the thermal energy of the carriers with regard to the state separation. Instantaneous carrier relaxation within each QD is therefore applied. Once the QD ensemble is populated to the required average occupancy, each QD is known with a specified instantaneous occupancy in terms of carrier number and energetic state.

Subsequently [Fig. 1(c)], many-body effects are introduced to the emission properties of the microstates of the QDs. We assume that the QDs have the same oscillator strength (and hence recombination lifetime). An empirical renormalization shift and an additional homogeneous broadening $^{16-18)}$ are introduced to the gain/spontaneous emission of each QD based on its instantaneous occupancy. We assume a linear dependence on carrier density, which is as reasonable an approximation as more detailed theoretical analyses. ${ }^{17)}$ The ground state of the QD contributes only to 


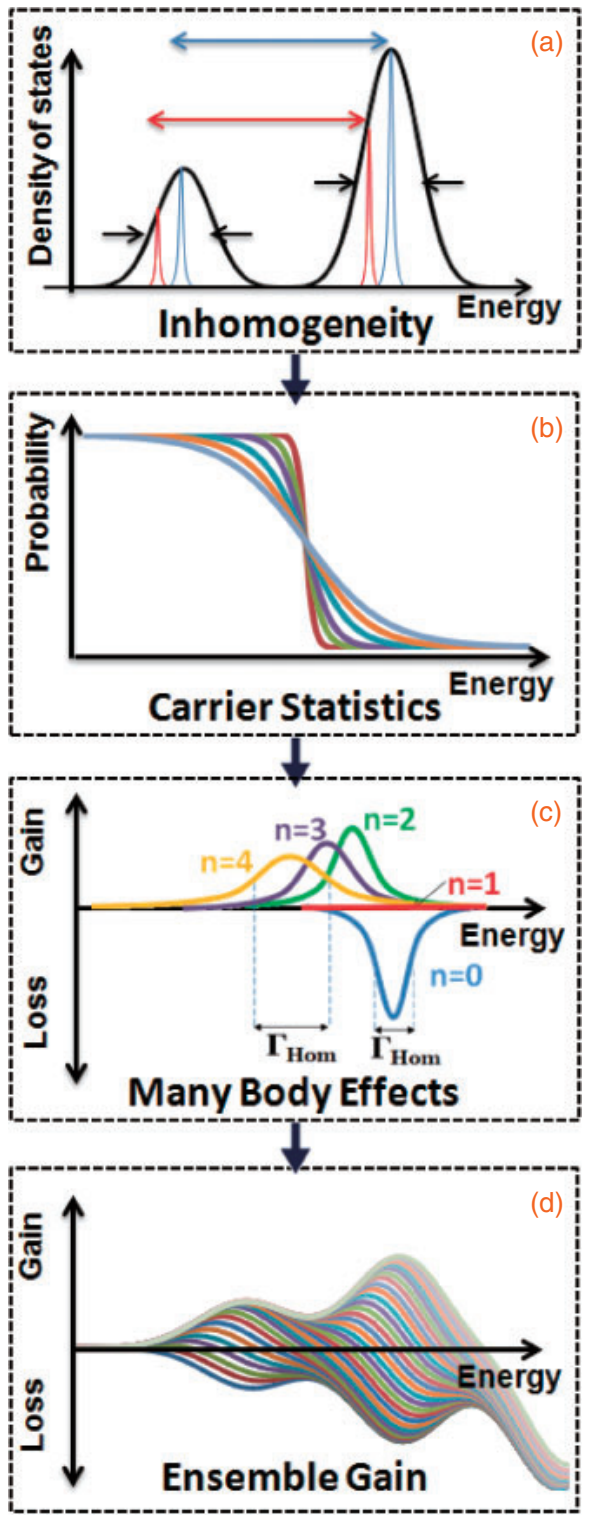

Fig. 1. Schematic of the approach to determining the gain spectra of QD lasers. (a) Ensemble of QDs with a Gaussian envelope for ground-state energy distribution. (b) Applying carrier statistics to the ensemble of QD states. (c) Applying many-body effects. (d) Gain calculation.

absorption at no carrier occupancy and would be transparent with one electron-hole (e-h) pair. Gain is obtained with two $\mathrm{e}-\mathrm{h}$ pairs in the QD. Many-body effects are manifested by an additional broadening and a renormalization shift to the transition energy. This shift and broadening continue as more carriers are added to the QD, owing to the continuously increasing dephasing yet saturated GS gain. We considered the homogeneous linewidth with no carriers to be $5 \mathrm{meV}$ and homogeneous broadening dependent on carrier density of $1 \mathrm{meV}$ per carrier per QD, as observed experimentally ${ }^{15)}$ at room temperature. The bandgap shrinkage is calculated using the bulk value of $32 \mathrm{meV} /\left(10^{18} \mathrm{~cm}^{-3}\right)^{1 / 3},{ }^{19)}$ which is in good agreement for both bulk and quantum well materials. ${ }^{20-22)}$ A QD volume of $10 \times 10^{-18} \mathrm{~cm}^{-3}$ was used. The resultant shifts in individual QD gain and SE peaks are in line with calculations $^{16-18)}$ and experimental observations. ${ }^{15)}$

In this paper, we describe simulation results that explore the effects of this free-carrier-induced shift and broadening on the carrier distribution function and then compare them with

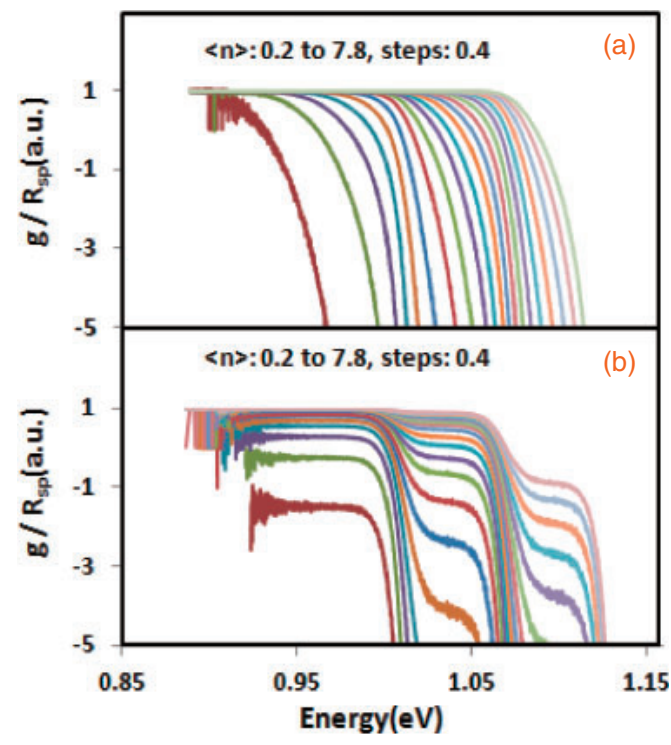

Fig. 2. Ratio of gain over spontaneous emission rate of Ensemble A of quantum dots at $300 \mathrm{~K}$ for (a) Fermi-Dirac and (b) random carrier distributions. $\langle n\rangle$ is the average number of carriers per dot.

experimental data. The different extreme cases for carrier statistics (Fermi-Dirac and random carrier distributions), QD ensemble inhomogeneity, and state separation are explored. We show that free carrier effects are not only important at high QD occupancies ${ }^{12)}$ but also at lower carrier densities where QD lasers would normally operate. We conclude that the free carrier effects dominate the form of the gain and spontaneous emission spectrum for the QD ensemble, and hence the carrier distribution function, rather than the carrier statistics.

Figure 2 shows simulation results of the ratio of gain to spontaneous emission $\left(P_{\mathrm{m}}\right)$ for an ensemble of quantum dots with an inhomogeneous linewidth of $41 \mathrm{meV}$ and a state separation of $60 \mathrm{meV}$ (Ensemble A) for (a) Fermi-Dirac and (b) random carrier distributions at room temperature. These values for state separation and inhomogeneous linewidth were measured for the QD laser material ${ }^{23}$ ) typical of the first generation of $1300 \mathrm{~nm}$ QD lasers. ${ }^{24)}$ The influence of free carrier effects is not considered in this data.

For Fermi-Dirac statistics [Fig. 2(a)], the distribution is a function of energy (QD size) with a priority for deeper confined dots due to the confinement energy dependence of carrier escape. By contrast, for random carrier statistics [Fig. 2(b)], rapid/continuous carrier escape and capture are not included, and all the QDs have the same carrier distribution function. The influence of the density of states on the probability distribution of carriers results in a steplike transition profile from the ground state (GS) to the first excited state (ES1) for both Fermi-Dirac and random carrier statistics, which is influenced by the state separation and inhomogeneous linewidth of the ensemble. Regions of the spectrum where GS and ES overlap occurs result in a modulation of the family of curves generated (e.g., $\sim 1 \mathrm{eV}$ ).

We now introduce an empirical value for the free-carrierinduced shift and additional homogeneous broadening of individual QD gain and SE as a function of carrier occupancy. ${ }^{15,25)}$ By incorporating these values into the model, the gain and spontaneous emission spectra were 


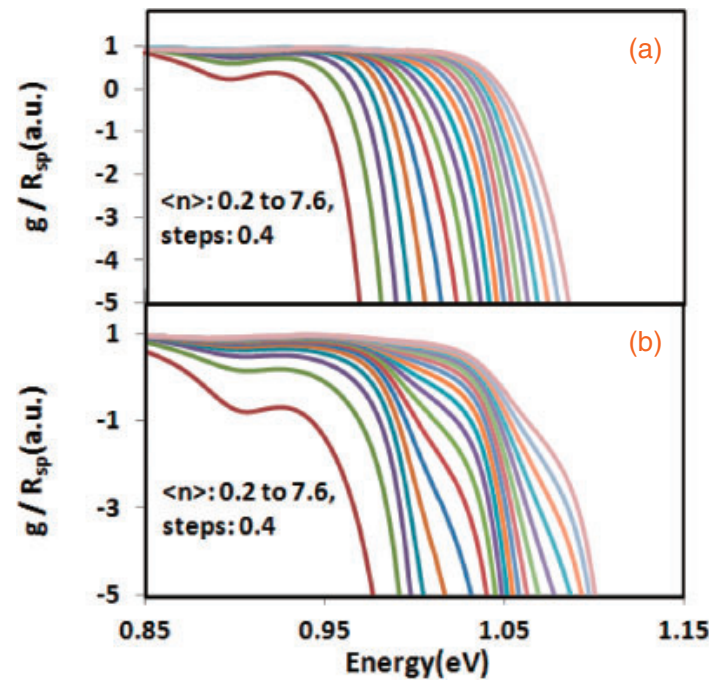

Fig. 3. Ratio of gain over spontaneous emission rate of Ensemble A of quantum dots at $300 \mathrm{~K}$ with free carrier effects for (a) Fermi-Dirac and (b) random carrier distributions. $\langle n\rangle$ is the average number of carriers per dot.

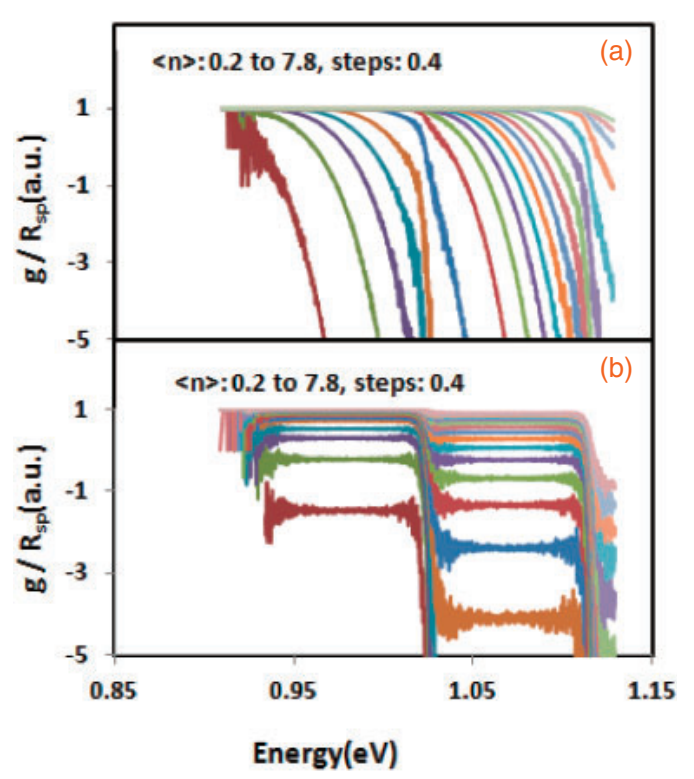

Fig. 4. Ratio of gain over spontaneous emission rate of Ensemble A for (a) Fermi-Dirac and (b) random carrier statistics at $300 \mathrm{~K}$.

recalculated, and the ratio of modal gain to spontaneous emission $\left(P_{\mathrm{m}}\right)$ is plotted for (a) Fermi-Dirac and (b) random carrier statistics at $300 \mathrm{~K}$ for Ensemble A. The addition of these free-carrier effects is significant and modifies these plots as compared with Fig. 2, where they are not included. This function is now seen to be very similar in both extreme cases of carrier statistics. Furthermore, a characteristic positive slope region is created in both data sets $(\sim 0.91 \mathrm{eV})$.

To explore the influences of QD state separation and inhomogeneous linewidth on the distribution function, we calculate the carrier distribution function for an ensemble of QDs with an inhomogeneous linewidth of $31 \mathrm{meV}$ and a state separation of $90 \mathrm{meV}$, termed Ensemble B. Results are plotted in Fig. 4(a) for a Fermi-Dirac distribution and in Fig. 4(b) for a random carrier distribution without the free carrier effects being applied to the model. These values

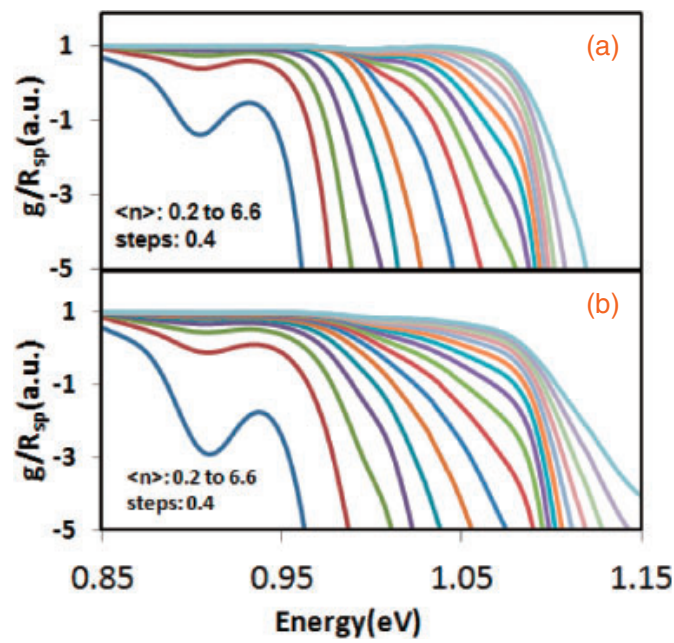

Fig. 5. Ratio of gain over spontaneous emission rate of Ensemble B of quantum dots at $300 \mathrm{~K}$ with free carrier effects for (a) Fermi-Dirac and (b) random carrier distributions. $\langle n\rangle$ is the average number of carriers per dot.

are typical for commercial, high-areal-density, and highuniformity QD laser material. ${ }^{26)}$

We observe that by using Fermi-Dirac statistics, the distribution is modulated in a much more abrupt manner in the transition region between the ground and excited states in comparison with Ensemble A. We also note that in the case of random carrier statistics, the flat carrier distribution signature is more clearly resolved at higher energies than that of Ensemble A.

We now apply free carrier effects to the model for the same ensemble of QDs using the same empirical values as applied to Ensemble A in Fig. 3. Figure 5 shows the results calculated for Ensemble B after inducing the free carrier effects. We note a more pronounced region of the spectra with a positive slope, but note once more that the two sets of data are very similar in form.

Figure 6 shows experimental data obtained from a QD laser with state separation and inhomogeneous linewidth as described for Ensemble B. ${ }^{23)}$ The device was a short-cavity $(250 \mu \mathrm{m})$, single-mode ( $3 \mu \mathrm{m}$ ridge width) laser diode and was maintained at a constant junction temperature of $300 \mathrm{~K}^{12)}$ The gain and spontaneous emission spectra of the laser were measured by the Hakki-Paoli technique. ${ }^{27)}$ We truncate the graph of g/Rsp [Fig. 6(c)] in regions where noise in the gain spectrum measurement dominates $(<\sim 0.916 \mathrm{eV})$.

Strong similarities are observed between the two simulations using very different carrier statistics [Figs. 5(a) and 5(b)], and this experimental data. The positive gradient region to the low-energy side of the ground state of the quantum dots (at $\sim 0.92 \mathrm{eV}$ in Figs. 3 and 5) is a particular signature of the effects of the free carriers. The poor signal-to-noise ratio in the long-wavelength region made resolving the dip observed in Fig. 5 impossible in this measurement.

We note that such a positive gradient was observed previously and attributed to a nonequilibrium carrier distribution. ${ }^{6)}$ However, this feature is present in our simulations owing to the inclusion of free carrier effects rather than the choice of carrier statistics. We highlight that the measured $P_{\mathrm{m}}$ does not resemble either of the plots in Fig. 4 where free carrier effects are ignored. 


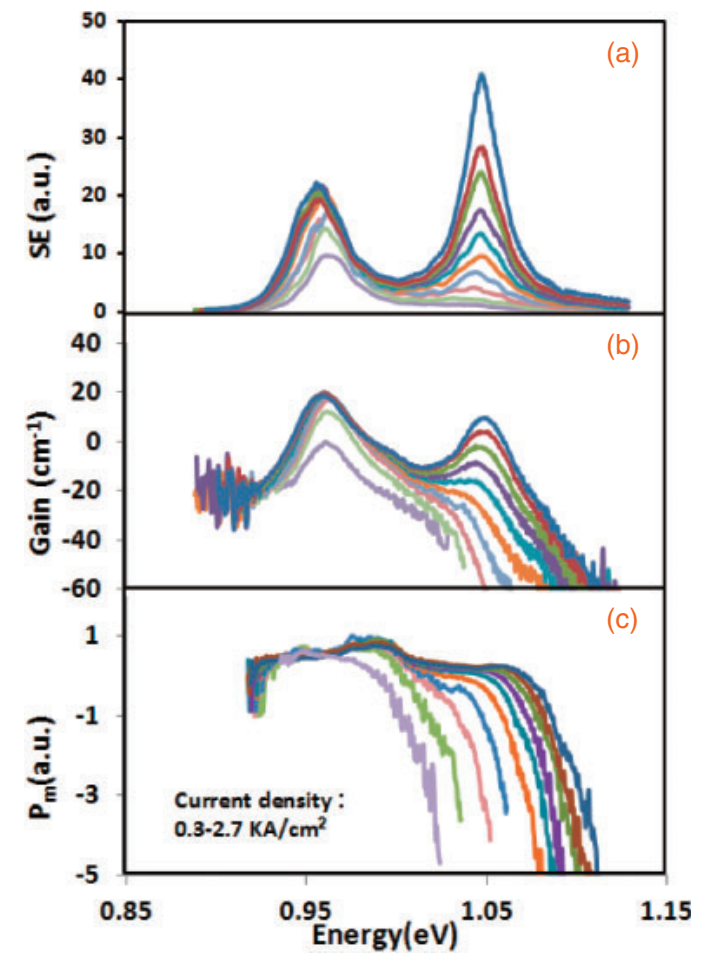

Fig. 6. Experimental results from Ensemble B. (a) Spontaneous emission, (b) gain, and (c) ratio of gain over spontaneous emission as a function of energy.

We note that differences between the simulated and experimental results are observed $(\sim 0.97 \mathrm{eV})$. We attribute this to the simulation not including any nominally forbidden transitions due to parity conservation (e.g., $\mathrm{e}_{1} \mathrm{hh}_{2}$ ). However, this approximation is not likely to be accurate since the QD is highly strained and the confinement potential is finite and parabolic. Indeed, a significant SE intensity is observed in the spectral region between the GS and ES ensembles, which represent a considerable residual if we consider a Gaussian fit to the ensemble of GS and ES states. We also note a feature in the gain spectrum at $\sim 1.0 \mathrm{eV}$ in Fig. 6(b), which may be due to a higher order transition (e.g., e1hh3 and e1hh5) with nonzero oscillator strength.

In this paper, we described the simulation results of the effects of free-carrier-induced shift and broadening on the carrier distribution function using a Monte Carlo model. Different carrier statistics (Fermi-Dirac and random carrier distributions) have been applied and compared. We show that the introduction of empirical values for the free-carrierinduced shift and broadening of the individual QD transitions results in very similar carrier distribution functions. We concluded that the free carrier effects have more influence on the form of gain and spontaneous emission spectrum for the QD ensemble than carrier statistics. This in turn suggests that carrier dynamics that determine the carrier statistics are of less importance than the carrier effects in influencing the form of gain and spontaneous emission spectrum. We compare our simulations with experimental data and confirm that a good fit is obtained for simulations incorporating free carrier effects with either extreme case for carrier statistics.

Acknowledgments We gratefully acknowledge the supply of QD laser material by QD Laser Inc. (Japan). We also acknowledge support from EPSRC under project EP/I018328/1.

1) Y. Arakawa and H. Sakaki, Appl. Phys. Lett. 40, 939 (1982).

2) M. Asada, Y. Miyamoto, and Y. Suematsu, IEEE J. Quantum Electron. 22, 1915 (1986).

3) N. Kirstaedter, N. N. Ledentsov, M. Grundmann, D. Bimberg, V. M. Ustinov, S. S. Ruvimov, M. V. Maximov, P. S. Kop'ev, Zh. I. Alferov, U. Richter, P. Werner, U. Gosele, and J. Heydenreich, Electron. Lett. 30, 1416 (1994).

4) D. L. Huffaker, G. Park, Z. Zou, O. B. Shchekin, and D. G. Deppe, Appl. Phys. Lett. 73, 2564 (1998).

5) M. A. Majid, D. T. D. Childs, K. Kennedy, R. Airey, R. A. Hogg, E. Clarke, P. Spencer, and R. Murray, Appl. Phys. Lett. 99, 051101 (2011).

6) H. D. Summers, J. D. Thomson, P. M. Smowton, P. Blood, and M. Hopkinson, Semicond. Sci. Technol. 16, 140 (2001).

7) M. Grundmann and D. Bimberg, Jpn. J. Appl. Phys. 36, 4181 (1997).

8) M. Grundmann and D. Bimberg, Phys. Rev. B 55, 9740 (1997).

9) M. Hutchings, I. O'Driscoll, P. M. Smowton, and P. Blood, Appl. Phys. Lett. 104, 031103 (2014).

10) A. Markus, M. Rossetti, V. Calligari, J. X. Chen, and A. Fiore, J. Appl. Phys. 98, 104506 (2005).

11) I. O'Driscoll, P. Blood, and P. M. Smowton, IEEE J. Quantum Electron. 46, 525 (2010).

12) H. Shahid, D. T. D. Childs, B. J. Stevens, and R. A. Hogg, Appl. Phys. Lett. 99, 061104 (2011).

13) N. Peyvast, H. Shahid, R. A. Hogg, and D. T. D. Childs, Appl. Phys. Express 8, 122102 (2015).

14) M. Lorke, T. R. Nielsen, J. Seebeck, P. Gartner, and F. Jahnke, Phys. Rey. B 73, 085324 (2006).

15) K. Matsuda, K. Ikeda, T. Saiki, H. Saito, and K. Nishi, Appl. Phys. Lett. 83, 2250 (2003).

16) H. C. Schneider and W. W. Chow, Phys. Rev. B 64, 115315 (2001).

17) S. V. Nair and Y. Masumoto, Phys. Status Solidi A 178, 303 (2000).

18) M. Lorke, W. W. Chow, T. R. Nielsen, J. Seebeck, P. Gartner, and F. Jahnke, Phys. Rev. B 74, 035334 (2006).

19) L. A. Coldren and S. W. Corzine, Diode Lasers and Photonic Integrated Circuits (Wiley, New York, 1995) p. 136.

20) J. Camassel, D. Auvergne, and H. Mathieu, J. Appl. Phys. 46, 2683 (1975)

21) S. Tarucha, H. Kobayashi, Y. Horikoshi, and H. Okamoto, Jpn. J. Appl. Phys. 23, 874 (1984).

22) D. A. Kleinman and R. C. Miller, Phys. Rev. B 32, 2266 (1985).

23) P. D. L. Greenwood, D. T. D. Childs, K. Kennedy, K. M. Groom, M. Hugues, M. Hopkinson, R. A. Hogg, N. Krstajić, L. E. Smith, S. J. Matcher, M. Bonesi, Sh. MacNeil, and R. Smallwood, IEEE J. Sel. Top. Quantum Electron. 16, 1015 (2010).

24) H. Y. Liu, D. T. Childs, T. J. Badcock, K. M. Groom, I. R. Sellers, M. Hopkinson, R. A. Hogg, D. J. Robbins, D. J. Mowbray, and M. S. Skolnick, IEEE Photonics Technol. Lett. 17, 1139 (2005).

25) R. Heitz, F. Guffarth, I. Mukhametzhanov, M. Grundmann, A. Madhukar, and D. Bimberg, Phys. Rev. B 62, 16881 (2000).

26) K. Nishi, T. Kageyama, M. Yamaguchi, Y. Maeda, K. Takemasa, T. Yamamoto, M. Sugawara, and Y. Arakawa, J. Cryst. Growth 378, 459 (2013).

27) B. W. Hakki and T. L. Paoli, J. Appl. Phys. 46, 1299 (1975). 